\title{
A conceptual system design study for an NBI beamline for the European DEMO
}

\author{
C. Hopf, G. Starnella, N. den Harder, B. Heinemann, U. Fantz \\ Max-Planck-Institut für Plasmaphysik, Boltzmannstr. 2, 85748 Garching bei München, Germany
}

\begin{abstract}
Neutral Beam Injection (NBI) as a robust, established heating and current drive (CD) method is considered for the European DEMO. Like on ITER, an NBI system for DEMO will have to use negative ions as the neutralisation efficiency of positive ions vanishes at the required $\geq 800 \mathrm{keV}$ beam energy. The required specifications of an NBI on DEMO go significantly beyond those on ITER, particularly when NBI is chosen to provide bulk current drive for a steady-state tokamak DEMO. The NBI beamline's power efficiency, on ITER only about $27 \%$, needs to be significantly increased to arrive at a tolerable recirculating power fraction. Envisaged solutions are the addition of energy recovery for the residual ions to a gas neutraliser or the replacement of the gas neutraliser by a photoneutraliser or a beam-driven plasma neutraliser. None of these concepts has been demonstrated on a relevant scale. In this article we outline our approach to a comprehensive system design study in order to explore a broad range of options for each beamline component and their mutual dependences, and we focus on the central role that the choice of the neutraliser concept - where needed in combination with energy recovery - plays for the layout of the whole beamline.
\end{abstract}

Keywords: neutral beam injection, DEMO, efficiency

\section{Introduction}

The European Roadmap to the Realisation of Fusion Energy [1] defines DEMO as the single step between ITER and a commercial Fusion Power Plant (FPP) with the mission to demonstrate energy production for the grid, a closed fuel cycle, and the readiness of the required technologies. Several different general design options for a DEMO are being discussed. If DEMO is going to be a tokamak these are a conservative pulsed device (also known as DEMO1), a steady-state tokamak (DEMO2) [2], and a machine that could be operated in both pulsed and steady-state mode, depending on the achieved $\mathrm{H}$ factor (FlexiDEMO) [3]. Neutral Beam Injection is one of the heating and current drive systems under consideration. Like the NBI on ITER [4], NBI for DEMO will also have to start from negative ions. Hence, return of experience from the ITER NBI beamlines and the test facilities preceding them is of high relevance.

DEMO1, not designed for a non-inductive scenario, has no explicit requirements for bulk current drive and is assumed to require a heating power of $\approx 50 \mathrm{MW}$ in steady operation, mostly for burn control and mode stabilisation [2]. Conversely, DEMO2 is designed to achieve fully non-inductive current drive. Assuming Neutral Beam Injection as the current drive system, the predictions for the required $\mathrm{CD}$ power range from 135 to $210 \mathrm{MW}$ [5] at a beam energy well in excess of the $1 \mathrm{MeV}$ on ITER. The requirements for a Flexi-DEMO will probably be similar. With such high powers continuously needed, the energy efficiency of the current drive system becomes an important issue. The required wall plug efficiency (injected power per total system power uptake) for DEMO2 or a fusion power plant is commonly cited as $\geq 55 \%$ [6]. This is by far higher than the wall plug efficiency of ITER's NBI that

\begin{tabular}{lcc}
\hline Efficiency & ITER NBI [7] & DEMO [8] \\
\hline Power supplies & 0.89 & 0.90 \\
Accelerator & 0.70 & 0.85 (target) \\
Neutralisation & 0.55 & 0.80 (target) \\
Duct transmission & 0.80 & 0.90 (target) \\
\hline Wall plug efficiency & 0.27 & 0.55 \\
\hline
\end{tabular}

Table 1: Energy efficiencies of the major beamline subsystems as expected for the ITER NBI and the corresponding values as a tentative set of targets for a steady-state DEMO, in order to achieve a wall-plug efficiency of 0.55 .

is expected to be around $27 \%$ [7]. Table 1 shows the energy efficiency of the whole ITER NBI beamlines and separately for its major components, and in the right column it shows reasonable target values for the component values that would lead to the desired wall plug efficiency.

The losses in the accelerator are dominated by the stripping of negative ions before full acceleration and the backacceleration of positive ions formed from background gas. In the beam duct losses are mostly due to beam scraping and reionisation. Both transmission efficiencies could be improved by a reduction of the background gas pressure as well as improved beam optics to decrease the beamlets divergence. The major limitation to the ITER NBI's efficiency, however, comes from the limited efficiency of its gas neutraliser. Promising ways to move beyond its efficiency are photo-neutralisation, gas neutralisation with energy recovery (ER), or plasma neutralisation. However, none of these options has been proven to date on a meaningful scale.

Unlike a steady-state DEMO, the pulsed DEMO1 might do with an efficiency that is only slightly improved from the ITER 


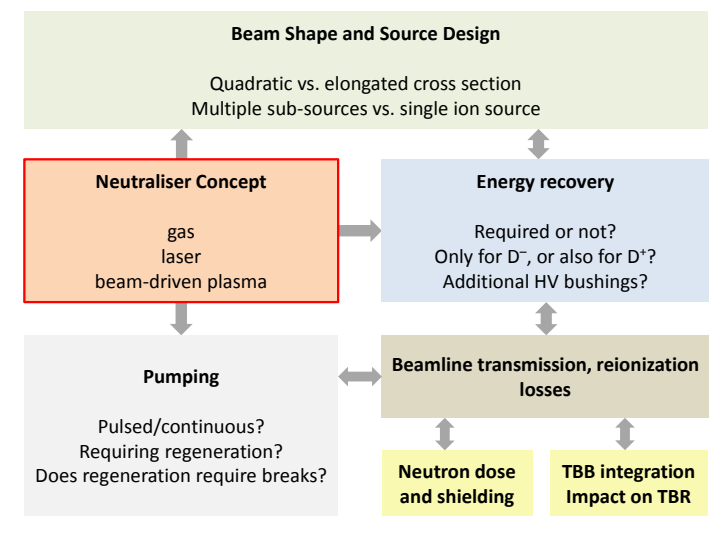

Figure 1: Schematic highlighting some of the important interdependences between different beamline components and their performance requirements. The neutraliser plays a key role. ( $\mathrm{HV}=$ high voltage, $\mathrm{TBB}=$ tritium breeding blanket, $\mathrm{TBR}=$ tritium breeding ratio)

value by conservative means. Being also the current baseline design, DEMO1 is also the machine that the detailed NBI development focusses on [7]. This approach is also in line with the roadmap [1], which states that DEMO should rely on "simple and robust technical solutions", and that "technologies desirable for advanced fusion power plants and as risk reduction elements, but not mature enough to be incorporated in DEMO, will have to be pursued in parallel". However, it can also be argued that choosing NBI for even a pulsed DEMO will only be a wise decision if there are at least credible concepts how the requirements of an advanced FPP could be met at a later stage. Hence, system studies that explore a broad range of beamline designs and technology combinations are needed to complement the detailed design study for a baseline NBI.

In this article we describe our approach to studying a broad range of beamline options. We will highlight the central role of the neutraliser in determining the requirements for the other components and, as a first result of our study, discuss the most attractive operational points when using different neutraliser choices.

\section{Approach to the study}

Our study rests on three columns:

- A simple system code that captures the essential physics of all beamline components by basic models and propagates a beam of extracted ions through the whole beamline, tracking their species evolution. One of the outputs of the code is the efficiency of the beamline, considering all reactions with the background gas and all acceleration and deceleration processes of both primary and secondary charges. This code serves to identify the sweet spots in the design and operational spaces and helps us make the selection which beamlines to study in more detail.

- The development of CAD assembly drawings that define the actual geometry and spatial arrangement of the beamline components.

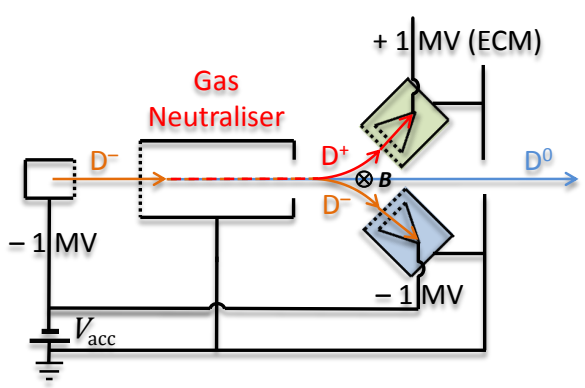

Figure 2: Schematic of energy recovery in combination with a gas neutraliser for both negative and positive ions. Power supplies that bias the ion collectors slightly to ensure complete capture of the residual ions and to avoid excessive lateral widening of the deceleration beam have been omitted for simplicity.

- Detailed three-dimensional beam optics and transport calculations, using tools that have already been successfully applied to the ASDEX Upgrade NBI [9, 10] and the baseline-DEMO NBI beamline [10].

\section{The neutraliser as key element}

ITER's gas neutraliser poses the major limitation to its NBI's energy efficiency. Hence, improving the neutraliser is key to enhancing the wall plug efficiency. Promising ways to do this are photoneutralisation, which promises theoretically unlimited neutralisation efficiency, recovery of the kinetic energy of the residual (i. e. non-neutralised) ions, and the (beam-driven) plasma neutraliser. The choice of the neutraliser is also a central decision as it influences the requirements for the other beamline components and their performance to a large extent. This is schematically shown in Fig. 1. The dependences are explained step by step in the remainder of this section.

Photo-(laser-)neutralisation was proposed for the DEMO NBI beamline $[11,12]$ but only demonstrated at small scale far from the parameters required for neutral beam heating [13, 14]. The idea is to pass the negative ion beam through an intense laser beam in an optical cavity with the laser wavelength chosen such that the photons can detach the loosely bound second electron, but cannot further ionize the resulting hydrogen atom. Hence, the neutralisation efficiency is theoretically unlimited and in practice efficiencies in excess of $90 \%$ are deemed achievable. Both resonant [11, 13] and non-resonant [12, 14] cavities for trapping the laser beam have been proposed and proof-of-principle tested. Besides the high neutralisation efficiency an additional significant advantage is that, as opposed to a gas or plasma neutraliser, a photoneutraliser does not introduce additional background gas into the beamline, reducing (re)ionisation and stripping losses and relaxing the requirements for the pumping system. As the laser beam and the ion beam have to have full overlap, and as the width of a laser beam in the optical cavity is limited, the ion beam's cross section should have a large aspect ratio (beam blade) [11, 7], with ramifications for the source design. However, while the physics are 


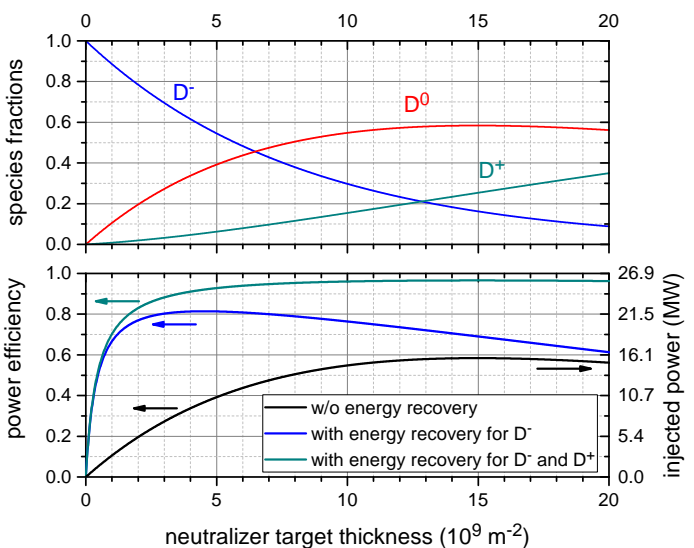

Figure 3: Top panel: Species evolution in the gas neutraliser as a function of gas target thickness. Bottom panel: maximum power efficiency, excluding any additional losses, without energy recovery (black), with energy recovery for $\mathrm{D}^{-}$ only, decelerated to $50 \mathrm{keV}$ (blue) and with eneregy recovery for negative and positive ions, both decelerated to $50 \mathrm{keV}$ (cyan). The black line can also be read as the injected power (right scale), assuming the current and beam energy of one ITER NBI beamline.

understood, the laser neutraliser's practical feasibility on the required scale and in the tokamak environment remains unclear.

Despite its limited efficiency, the gas neutraliser could still be an attractive option when used in conjunction with an energy recovery (ER) system [15]. It is schematically depicted in Fig. 2 and its practical application has been experimentally demonstrated for positive NBI ion beams [16]. Both negative (nonstripped fast ions) and positive ions (doubly stripped) exit the neutraliser along with the neutral beam. Recovery of their energy relies on deflecting negative and positive ions into different directions in a first stage and then electrostatically decelerating them before they hit the collector. Schematically this is simple for the negative ions, as the negative ion collector only has to be electrically connected to the ion source potential. A small power supply that provides a bias to the collector to ensure that all negative ions strike the collector and to prevent a lateral blow up of the decelerated beam is omitted in the schematic. The recovery of the positive ions is more complicated, as they have to be collected on a potential that is twice the total acceleration voltage away from the ion source and this energy has to be converted into useful electrical energy. Devices called energy conversion modules (ECM) have been developed for the purpose and proven the principle.

The benefit of ER in combination with a gas neutraliser becomes clear from Fig. 3. While the fast negative hydrogen ions travel through the neutraliser they are stripped to neutral hydrogen and/or stripped further to fast positive ions. At the point in Fig. 3 where the fraction of neutral atoms, and with it the neutraliser's energy efficiency without ER, reaches its maximum of about $55 \%$, i.e. at the optimal target thickness, the fractions of negative and positive residual ions are almost equal. As Fig. 3 illustrates, when recovering the energy of the negative ions only, the energy efficiency can be increased to almost $80 \%$. However, the maximum of this efficiency is at lower target thickness where the neutralisation yield is consid- erably lower $(\sim 25 \%)$, meaning that despite a possibly good wall plug efficiency the injected power of such a system would be severely reduced (right scale). At the target thickness of the optimal neutralisation efficiency the energy efficiency gain is considerably smaller, but at intermediate target thicknesses an attractive balance between energy and neutralization efficiency can be found. When recovering the energy of both polarities of residual ions the efficiency remains above $80 \%$ up to the optimal neutralisation efficiency, therefore representing the operational sweet spot. The actually achievable energy efficiency can be lowered due to additional losses, e.g. by acceleration of secondary charges.

Due to the high potential differences between the collectors, their deceleration grids and the grounded surroundings the ER system shares much complexity with the accelerator. This means, that also the space demand will be considerable, most likely making the beamline longer. This may increase reionisation and transmission losses, reducing the net efficiency gain. From a beam optics perspective, designing an energy recovery system becomes the more difficult the larger the beam cross section or the more (sub)beams are installed on a single beamline. Hence, the energy recovery system puts constraints on the beam (source) shape.

The plasma-neutraliser can be thought of as an improved efficiency version of the gas neutraliser due to stripping of the negative ions' weakly bound electrons by collisions with the electrons in a dense low-temperature plasma in the neutraliser [17]. The challenge is the production of a sufficiently dense plasma, which, when done by coupling energy from external sources $[18,19]$, becomes energetically less attractive. To circumvent this problem, creation of the required plasma density (several $10 \%$ of the neutral gas density) by the beam itself and enhanced electron confinement due to cusp magnets was proposed and the performance was estimated with a zero-dimensional model $[20,21]$ that balances the energy input by the beam and the losses to the wall along the cusp lines.

We applied an own implementation of this model to a beam with constant ITER-like beam energy $(1 \mathrm{MeV})$ and the accelerated current density $\left(77 \mathrm{~A} / \mathrm{m}^{2}\right.$ like averaged over one beamlet group of the ITER NBI grids), but varying the beam current together with its cross sectional area (Fig. 4). As the beam drives the plasma formation and as its neutralisation efficiency depends on the plasma density, the neutralisation efficiency increases with beam power. The predicted efficiency for the full ITER beam is higher than $70 \%$. As the losses scale with the wall area, there is also a dependence on the aspect ratio of the neutraliser cross section. Fig. 4 shows a calulation where an ITER beam of full accelerated current travels through a plasma neutraliser of constant length $z$ and cross sectional area $x \times y$, while varying the aspect ratio $y / x$ of beam and neutraliser. It is obvious that the square shape produces the highest efficiency. This is a distinct difference in comparison with both the photoneutraliser, which needs to have a large aspect ratio for overlap with the laser, and the gas neutraliser, where narrow, i.e. high aspect ratio, channels are useful to keep the gas inflow for a certain gas density as low as possible.

A potentially harmful effect on the beam quality of the 


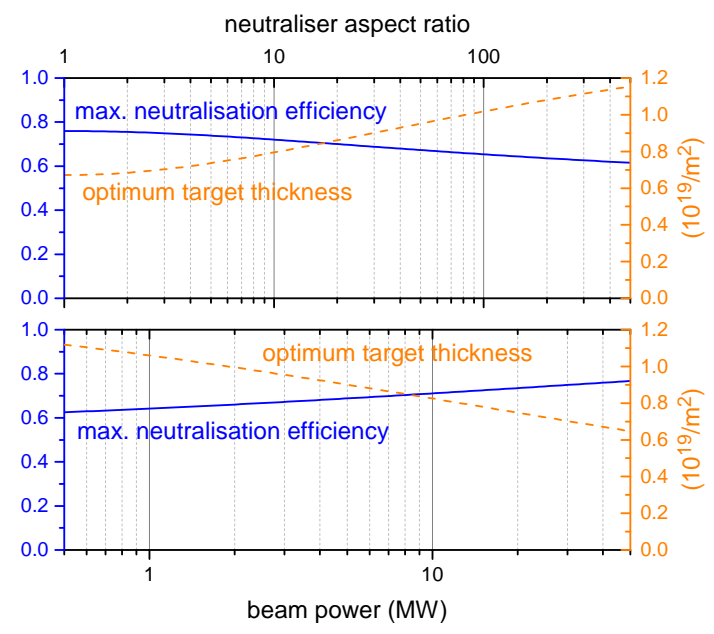

Figure 4: Maximum neutralisation efficiency (solid blue) of the beam-driven plasma neutraliser at the optimal target thickness (dashed orange) as a function of beam power (bottom) and aspect ratio of the beam and neutraliser cross section (top). The assumed beam parameters were $1 \mathrm{MeV} \mathrm{D}^{-}$with a constant beam current density of $77 \mathrm{~A} / \mathrm{m}^{2}$ (ITER's specified current density averaged over the area of one beamlet group) and the beam and neutraliser cross sections were chosen according to the total current. For the beam power dependence the assumed aspect ratio was one.

plasma neutraliser comes from the ion beam deflection induced by the electron-confining magnetic field that needs to cross the beam at the entrance (and the exit) of the neutraliser.

Due to the different amount of gas injected, the choice of the neutraliser principle has a pronounced influence on the required pumping speed and technology, and both neutraliser principle and pumps determine the beamline's background pressure and associated beam losses. The choice of high-speed pumps for an NBI system working with very long to quasi infinite pulses is a challenge in its own right. While current NBI systems often use large area cryopumps or other getter pumps, such pumps require cyclic regeneration that is probably not compatible with the continuous operation. Mercury diffusion pumps as suggested for the DEMO divertor [22] might provide a viable alternative, albeit at lower pumping speed per unit area, demanding a reduction of the gas flow into the beamline.

There is also a requirement to keep the NBI port openings to the torus small and few in order to minimise the effect on the tritium breeding ratio [6] and to limit the neutron flux into the beamline that might lead to significant radiation damage to beamline components. The required port opening size is ultimately determined by the single beamlets' divergence and distance from source, if all beamlets are steered such that they intersect in a common point inside the opening in the tritium breeding blanket. Inclusion of energy recovery will make the beamline longer and either increase the required opening or increase the beam transport losses, reducing the overall efficiency gain through ER.

\section{Conclusion}

The NBI's wall-plug efficiency, on ITER chiefly limited by the maximum neutralisation efficiency of the gas neutraliser of $\sim 55 \%$, needs to be significantly increased for DEMO, particularly if DEMO is to demonstrate non-inductive steady-state operation using NBI as its main current drive system. Efficiency enhancement concepts exist, particularly regarding improved neutralisers, but their practical demonstration at relevant parameters cannot be expected in the near future. Hence, we are exploring a broad range of technology choices and combinations with the aim of identifying multiple credible strategies for beamlines with improved efficiency. As discussed in this article, the neutraliser and the energy recovery system, if needed, play a key role as they constrain many other design choices, such as the ion source and beam shape, the beamline length or the required pumping speed. In principle the gas neutraliser with energy recovery, the beam-driven plasma neutraliser, or the photoneutraliser all promise to achieve attractive energy efficiencies. However, secondary effects such as the influence of beamline length as well as feed gas flow and pumping have to be considered as well in order determine the implications of the choices on the wall-plug efficiency. While we estimate these dependences using a system of coupled simple physics models for the beamline components, the final stage of the study will be concrete layouts with CAD assembly drawing, accompanied with detailed 3D beam transport and background gas simulations.

\section{Acknowledgement}

This work has been carried out within the framework of the EUROfusion Consortium and has received funding from the Euratom research and training programme 2014-2018 under grant agreement No 633053. The views and opinions expressed herein do not necessarily reflect those of the European Commission.

[1] F. Romanelli (Ed.), Fusion Electricity - A roadmap to the realisation of fusion energy, EFDA, 2012.

[2] R. Wenninger, R. Kembleton, C. Bachmann, W. Biel, T. Bolzonella, S. Ciattaglia, F. Cismondi, M. Coleman, A. Donné, T. Eich, E. Fable, G. Federici, T. Franke, H. Lux, F. Maviglia, B. Meszaros, T. Pütterich, S. Saarelma, A. Snickers, F. Villone, P. Vincenzi, D. Wolff, H. Zohm, The physics and technology basis entering european system code studies for DEMO, Nucl. Fusion 57 (2017) 016011.

[3] H. Zohm, F. Truble, W. Biel, E. Fable, R. Kemp, H. Lux, M. Siccinio, R. Wenninger, A stepladder approach to a tokamak fusion power plant, Nucl. Fusion 57 (2017) 086002.

[4] R. S. Hemsworth, D. Boilson, P. Blatchford, M. D. Palma, G. Chitarin, H. P. L. de Esch, F. Geli, M. Dremel, J. Graceffa, D. Marcuzzi, G. Serianni, D. Shah, M. Singh, M. Urbani, P. Zaccaria, Overview of the design of the ITER heating neutral beam injectors, New J. Phys. 19 (2017) 025005 .

[5] G. Giruzzi, J. Artaud, M. Baruzzo, T. Bolzonella, E. Fable, L. Garzotti, I. Ivanova-Stanik, R. Kemp, D. King, M. Schneider, R. Stankiewicz, W. Stepniewsk, P. Vincenzi, D. Ward, , R. Zagorski, Modelling of pulsed and steady-state DEMO scenarios, Nucl. Fusion 55 (2015) 073002.

[6] T. Franke, P. Agostinetti, K. Avramidis, A. Bader, C. Bachmann, W. Bie, T. Bolzonella, S. Ciattaglia, M. Coleman, F. Cismondi, G. Granucci, G. Grossetti, J. Jelonnek, I. Jenkins, M. Kalsey, R. Kembleton, N. Mantel, J.-M. Noterdaeme, N. Rispoli, A. Simonin, P. Sonato, M. Tran, 
P. Vincenzi, R. Wenninger, Heating \& current drive efficiencies, TBR and RAMI considerations for DEMO, Fus. Eng. Des. 123 (2017) 495.

[7] P. Sonato, P. Agostinetti, T. Bolzonella, F. Cismondi, U. Fantz, A. Fassina, T. Franke, I. Furno, C. Hopf, I. Jenkins, E. Sartori, M. Tran, J. Varje, P. Vincenzi, L. Zanotto, Conceptual design of the DEMO neutral beam injectors: main developments and R\&D achievements, Nucl. Fusion 57 (2017) 056026.

[8] U. Fantz, C. Hopf, R. Friedl, S. Cristofaro, B. Heinemann, S. Lishev, A. Mimo, Technology developments for a beam source of an nnbi system for demo, Fus. Eng. Des. in press. doi:https://doi.org/10.1016/j.fusengdes.2018.02.025.

[9] N. den Harder, D. Rittich, G. Orozco, C. Hopf, the ASDEX Upgrade Team, Neutral beam injection at ASDEX Upgrade: transmission and beamline losses, EPS Conf. Proc. 41F.

[10] N. den Harder, in: this SOFT conference, 2018.

[11] A. Simonin, J. Achard, K. Achkasov, S. Bechu, C. Baudouin, O. Baulaigue, C. Blondel, J. Boeuf, D. Bresteau, G. Cartry, W. Chaibi, C. Drag, H. de Esch, D. Fiorucci, G. Fubiani, I. Furno, R. Futtersack, P. Garibaldi, A. Gicquel, C. Grand, P. Guittienne, G. Hagelaar, A. Howling, R. Jacquier, M. Kirkpatrick, D. Lemoine, B. Lepetit, T. Minea, E. Odic, A. Revel, B. Soliman, P. Teste, R\&D around a photoneutralizerbased NBI system (Siphore) in view of a DEMO tokamak steady state fusion reactor, Nucl. Fusion 55 (2015) 123020.

[12] A. Fassina, F. Pretato, M. Barbisan, L. Giudicotti, R. Pasqualotto, A feasibility study of a NBI photoneutralizer based on nonlinear gating laser recirculation, Rev. Sci. Instrum. 87 (2016) 02B318.

[13] D. Bresteau, C. Blondel, C. Drag, Saturation of the photoneutralization of $\mathrm{a} \mathrm{H}^{-}$beam in continuous operation, Rev. Sci. Instrum. 88 (2017) 113103.

[14] S. Popov, M. Atlukhanov, A. Burdakov, A. Ivanov, A. Kasatov, A. Kolmogorov, R. Vakhrushev, M. Y. Ushkova, A. Smirnov, A. Dunaevsky, Neutralization of negative hydrogen and deuterium ion beams using nonresonance adiabatic photon trap, Nucl. Fusion 58 (2018) 096016.

[15] R. McAdams, A. Holmes, M. Porton, A. Benn, E. Surrey, T. T. C. Jones, Advanced energy recovery concepts for negative ion beamlines in fusion power plants, AIP Conf. Proc. 1515 (2013) 559.

[16] M. Fumelli, F. Jequier, J. Paméla, M. Hanada, Y. Okumura, K. Watanabe, Energy recovery experiments with a powerful $100 \mathrm{keV}$ D-based neutral beam injector, AIP Conf. Proc. 287 (1992) 909.

[17] K. Berkner, Plasma neutralizers for $\mathrm{H}^{-}$or $\mathrm{D}^{-}$beams, in: Second International Symposium on the Production and Neutralization of Negative Ions and Beams, Upton, NY, October 6-10, 1980, 1980.

[18] V. Zhiltsov, E. Klimenko, P. Kosarev, V. Kulygin, V. Platonov, N. Semashko, A. Skovoroda, V. Ukhov, The development of a negative ion beam plasma neutralizer for ITER NBI, Nucl. Fusion 40 (2000) 509.

[19] M. Hanada, M. Kashiwagi, T. Inoue, K. Watanabe, T. Imai, Experimental comparison between plasma and gas neutralization of high-energy negative ion beams, Rev. Sci. Instrum. 75 (2004) 1813.

[20] E. Surrey, A. Holmes, The beam driven plasma neutralizer, AIP Conf. Proc. 1515 (2013) 532.

[21] I. Turner, Final report on deliverable feasibility of testing beam-driven plasma neutralisation on SNIF, Tech. rep., EUROfusion (2016).

[22] T. Giegerich, C. Day, Conceptuation of a continuously working vacuum pump train for fusion power plants, Fus. Eng. Des. 88 (2013) 2206. 